\title{
Current practice for diagnosing immediate drug hypersensitivity reactions in Korea
}

\author{
Sung-Yoon Kang ${ }^{1, *}$, Min-Suk Yang ${ }^{2,3,}$, , Woo-Jung Song ${ }^{4}$, and Sang-Heon Cho ${ }^{2}$
}

\begin{abstract}
${ }^{1}$ Department of Internal Medicine, Gachon University Gil Medical Center, Incheon; ${ }^{2}$ Department of Internal Medicine, Seoul National University College of Medicine, Seoul; ${ }^{3}$ Department of Internal Medicine, Seoul Metropolitan Government Seoul National University Boramae Medical Center, Seoul; ${ }^{4}$ Department of Allergy and Clinical Immunology, Asan Medical Center, University of Ulsan College of Medicine, Seoul, Korea
\end{abstract}

Received: April 7, 2020 Revised : May 18, 2020 Accepted: May 23, 2020

\section{Correspondence to}

Sang-Heon Cho, M.D.

Department of Internal

Medicine, Seoul National

University College of Medicine,

101 Daehak-ro, Jongno-gu, Seoul

03080, Korea

Tel: +82-2-2072-2971

Fax: +82-2-764-3954

E-mail: shcho@snu.ac.kr

https://orcid.org/0000-0002-

7644-6469

*'These authors contributed equally to this work.
Background/Aims: Skin (STs) and drug provocation (DPTs) tests are essential for identifying the culprit drugs causing drug hypersensitivity reactions (DHRs). Several protocols have been developed for the identification of some culprit drugs, but they are neither thoroughly validated nor standardized. Furthermore, language barriers may impede the exchange of information necessary for test standardization.

Methods: We searched the Korean literature for articles on drug hypersensitivity published from 1933 to 2016 using the KoreaMed search engine and archives of Korean journals. We reviewed and rated all articles according to the description of STs and DPTs.

Results: Of the 632 articles obtained in our initial search, 34 had adequate descriptions of 15 STs and 22 DPTs. Up to 27 healthy control subjects in STs were enrolled to determine non-irritating concentrations. The concentrations used for intradermal tests were commonly a $1 / 10$ dilution of those used for skin prick tests. The interpretations of the STs were mostly similar among researchers. For DPTs, most procedures were single-arm open-label tests of various drugs. The initial dose ranged from a quarter dose to a single therapeutic dose, depending on the severity of the original hypersensitivity reaction. The interval between doses was usually 30 to 60 minutes, and a positive reaction usually occurred within twice the time of the original reaction.

Conclusions: Efforts to distribute information are necessary to standardize protocols and better understand DHRs.

Keywords: Diagnosis; Drug hypersensitivity; Drug provocation test; Immediate hypersensitivity; Skin test

\section{INTRODUCTION}

An adverse drug reaction (ADR) is a noxious, unintended, or undesired reaction to a drug occurring at doses normally used for the prevention, diagnosis, or treatment of disease [1]. Drug hypersensitivity reactions (DHRs), which comprise $10 \%$ to $20 \%$ of ADRs, are a public health issue causing significant mortality, morbidity, and socioeconomic costs that are probably underesti- mated $[2,3]$. Because DHRs are difficult to predict, it is important to preclude re-exposure to the causative drug in patients who have developed symptoms. Hence, it is necessary to accurately identify the responsible drugs.

To identify the culprit drugs causing DHRs, especially type I (immunoglobulin E [IgE]-mediated) hypersensitivity to a drug, in vivo and in vitro tests based on thorough clinical history are essential [4,5]. Although in vitro tests such as the detection of drug-specific IgE and the 
basophil activation test are safe and convenient, they are available only for a few drugs, and many of them still need clinical validation [5]. Thus, in vivo tests are the mainstay for identifying the culprit drug causing a DHR. Compared with drug provocation tests (DPTs), skin tests (STs), such as skin prick and intradermal tests, are more commonly used in vivo because they are relatively safe and simple to perform [6]. For STs, it is necessary to establish the highest concentration of each drug that would not elicit an irritant skin reaction in normal subjects. It is also vital to interpret these tests in a standardized way to ensure that the results are the same, no matter where and when the test is performed. When the causative agents cannot be confirmed with these indirect tests or when indirect tests are not available, DPTs, the ultimate in vivo test, can be conducted. DPT is the gold standard for confirming the causative agents of ADRs [7]. DPTs are generally conducted using in-house protocols at each center, and the number of steps, concentration the drug administered at each step, and interval between steps need to be determined to create a protocol.

Recently, the European Network of Drug Allergy (ENDA) and the Drug Hypersensitivity interest group in the European Academy of Allergy and Clinical Immunology (EAACI) reviewed the literature to recommend protocols for in vivo tests for the diagnosis of DHRs $[6,7]$. Nevertheless, diagnostic procedures for many drugs have not been established because of the rarity of DHRs. In particular, numerous works on diagnostic protocols for DHRs have been published in languages other than English, which makes that information much less accessible to the wider world [6-9]. The increasing use of drugs has led to a substantial increase in hypersensitivity reactions, which also need to be managed within the framework for diagnostic protocols [5]. Based on this awareness, we reviewed the Korean literature on DHRs and summarized the protocols used in those studies for STs and DPTs.

\section{METHODS}

We searched the KoreaMed database (https://koreamed. org) for relevant studies published from January 1933 to December 2016. KoreaMed is a free search engine used to access articles published in Korean medical, dental, nursing, nutrition, and veterinary journals, provided by the Korean Association of Medical Journal Editors. We retrieved data using the following keywords: "drug anaphylaxis" OR "allergy" OR "hypersensitivity." We also searched the archives of the Korean Journal of Internal Medicine, the official journal of the Korean Association of Internal Medicine and Allergy Asthma \& Respiratory Disease and its predecessors, including Allergy (Seoul) and the Journal of Asthma, Allergy and Clinical Immunology, which are official journals of the Korean Academy of Allergy, Asthma and Clinical Immunology [10].

We reviewed all articles and selected articles detailing the methods of STs and DPTs for the diagnosis of an immediate hypersensitivity reaction. An immediate reaction was defined as a reaction that occurred within 6 hours after drug exposure with clinical features of typical type I hypersensitivity: urticaria, angioedema, bronchospasm, anaphylaxis, etc. [11,12]. The description of the ST method was considered to have adequate quality when all of the following were present: the name and concentration of the drug used in the ST, and the results in healthy controls. Articles with detailed descriptions of the DPT procedure, such as the doses at each step, interval between steps, and the result, were included in this study.

The initial search yielded 632 articles. After removing 427 papers based on the title and abstract, we evaluated 205 full-text articles. Fifty-five articles did not describe the diagnostic tests. Of the remaining 150, 116 were excluded because they did not describe the ST or DPT procedure sufficiently. Ultimately, 34 articles were included in this study. Three of the 34 articles provided adequate information on both STs and DPTs. The selection processes are outlined in Fig. 1.

All selection steps, including data extraction, inclusion eligibility, and quality assessment, were performed by two researchers independently. If the two did not agree, disagreements were resolved by reaching a consensus through discussion.

This study was approved by the Institutional Review Board of the Seoul Metropolitan Government Seoul National University Boramae Medical Center (IRB No. o72020-257). Informed consent was waived by the board. 


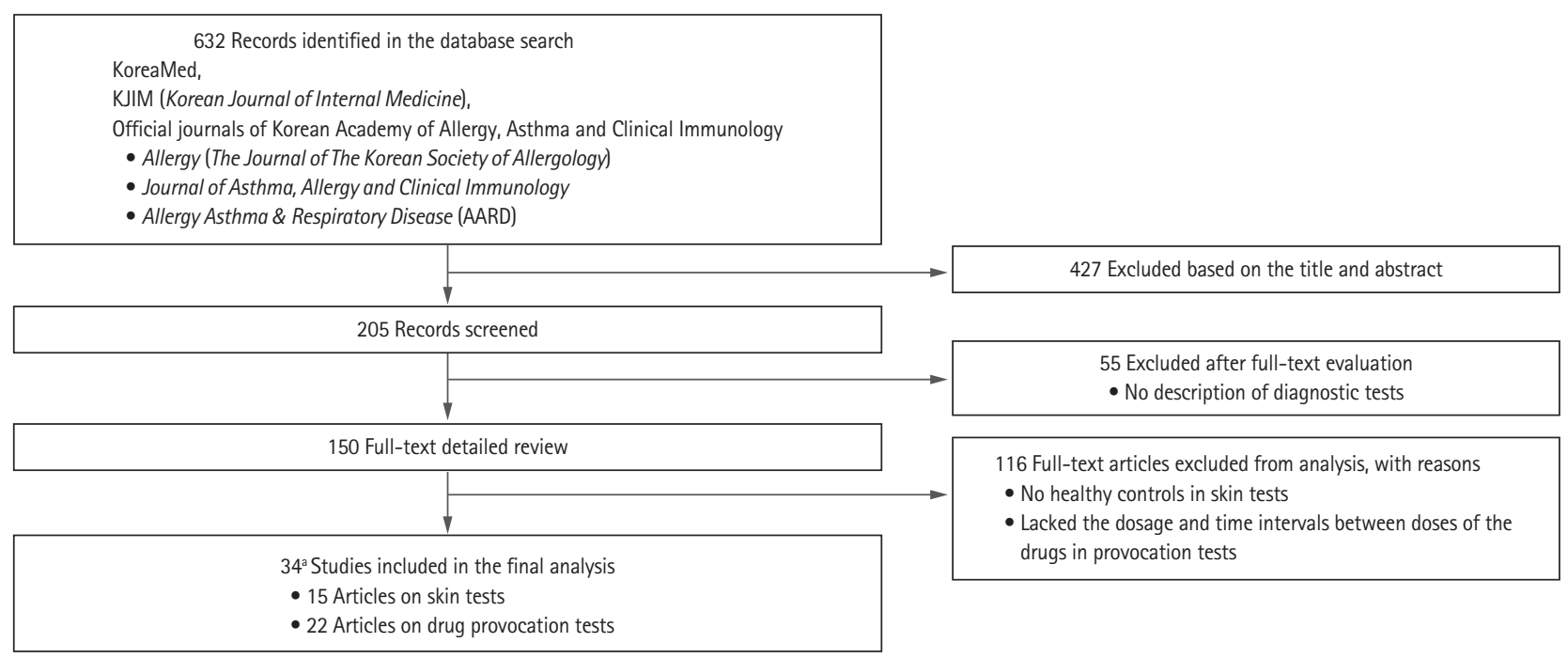

Figure 1. Flow chart of study selection. ${ }^{a}$ Of the 34 articles, three included both skin test and drug provocation test protocols.

\section{RESULTS}

\section{STs for the diagnosis of drug hypersensitivity}

We identified 15 studies using STs to identify culprit drugs [13-27]. STs for a variety of drugs, including antibiotics other than penicillin, $\mathrm{H} 2$ receptor antagonists, local anesthetics, leukotriene antagonists, antitussives, multivitamins, and hormones, were performed in 31 patients with suspected immediate allergic reactions, such as anaphylaxis, urticaria, and angioedema. In most studies, skin prick tests (SPTs) were performed initially, and intradermal tests (IDTs) were performed when the results of the SPTs were negative. To establish the non-irritant concentration (NIC), 3 to 27 healthy volunteers (mean, 10.3 \pm 6.5 subjects) were recruited in each study. The concentrations for IDT started at 1/1,oooth of the concentration used for the SPT depending on the drug; commonly used concentrations were 1/10th that used in the SPTs. For some drugs, IDTs were performed sequentially with increasing drug concentrations. In most studies, the SPT or IDT results were assessed using the absolute wheal size or the ratio of the wheal size induced by the test drug to that induced with a positive control. The criteria for positive IDT reactions were similar to those of SPTs (Table 1).

\section{DPTs for the diagnosis of drug hypersensitivity}

We identified 22 studies (18 studies reporting on 203 adults and four studies reporting on 74 children) on DPTs that were mostly performed as single-arm open-label tests for identifying culprit drugs [19,20,26,28-46]. DPTs in adults were performed with analgesics, antimicrobials, gastrointestinal medications, muscle relaxants, antitussives, $\mathrm{H} 1$ receptor antagonists, and corticosteroids. A subcutaneous challenge test was used with local anesthetics, such as lidocaine. The DPTs for pediatric patients involved analgesics, antimicrobials, antitussives, antiepileptic drugs, and lactose. The most common reason for DPTs was anaphylaxis, followed by urticaria, angioedema, skin eruption, and other symptoms, such as cough and dyspnea. For all drugs except aspirin, the initial dose of the DPT ranged from a quarter of the standard therapeutic dose to a single full therapeutic dose. DPTs were performed with lower initial concentrations in cases with a severe reaction, such as anaphylaxis. Further dosage increases were typically twice the previous dose until the standard therapeutic level was achieved. The interval between doses ranged from 30 minutes to 3 hours but was usually between 30 minutes and 60 minutes. A positive reaction, defined as the reproduction of symptoms, usually occurred within twice the time that the initial reaction took from exposure to the culprit drug (original latency period) (Table 2). DPT 


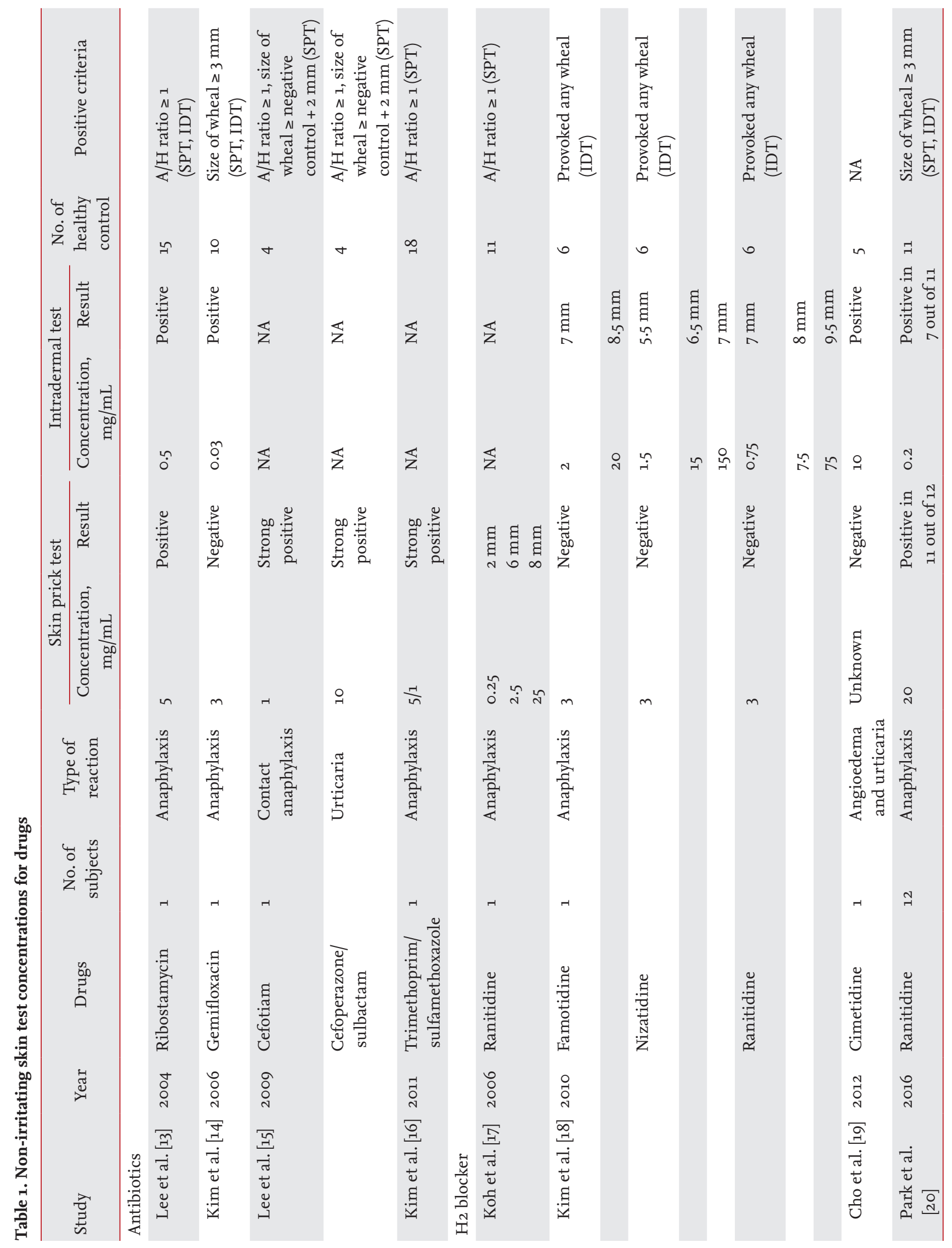




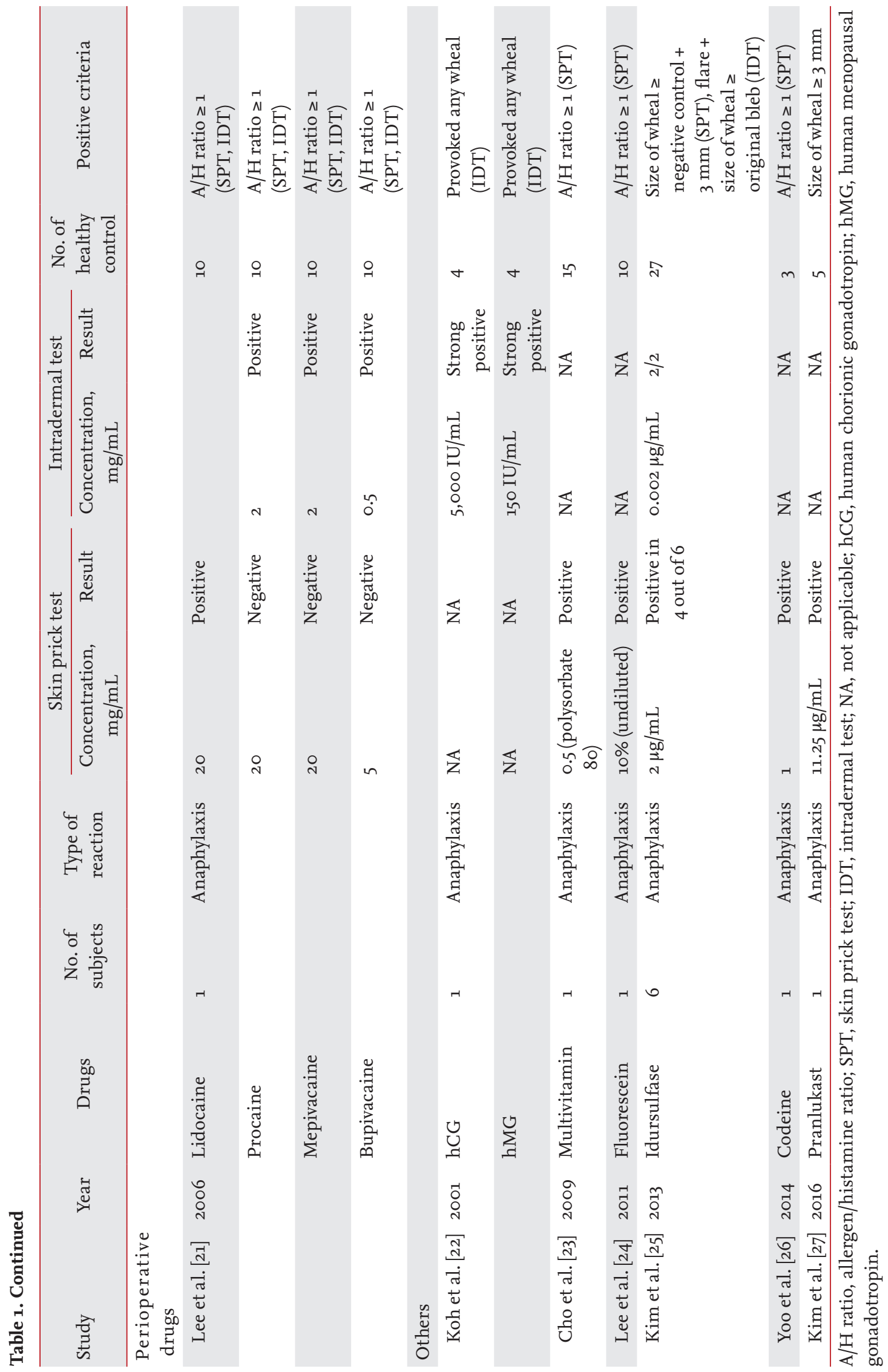




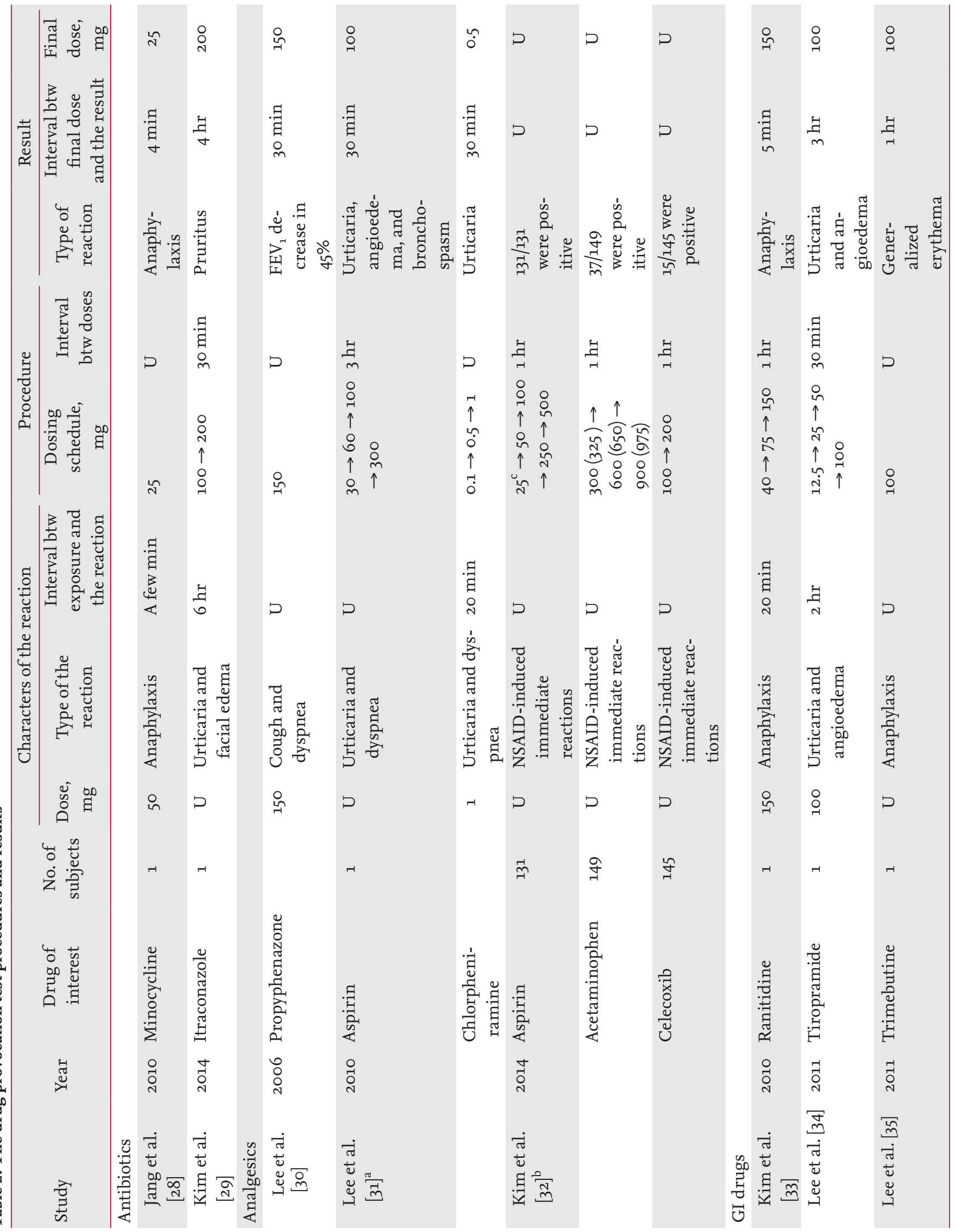




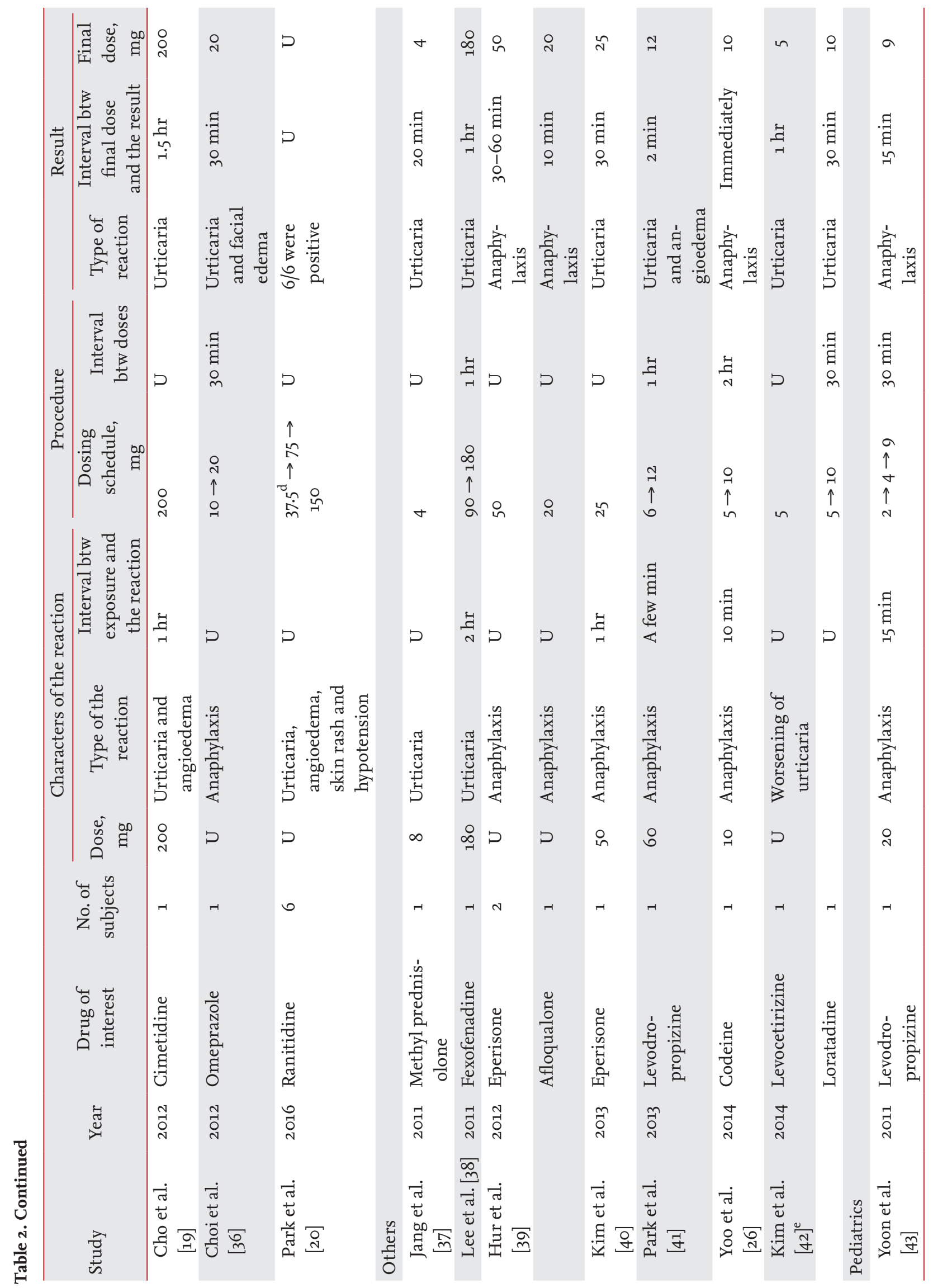




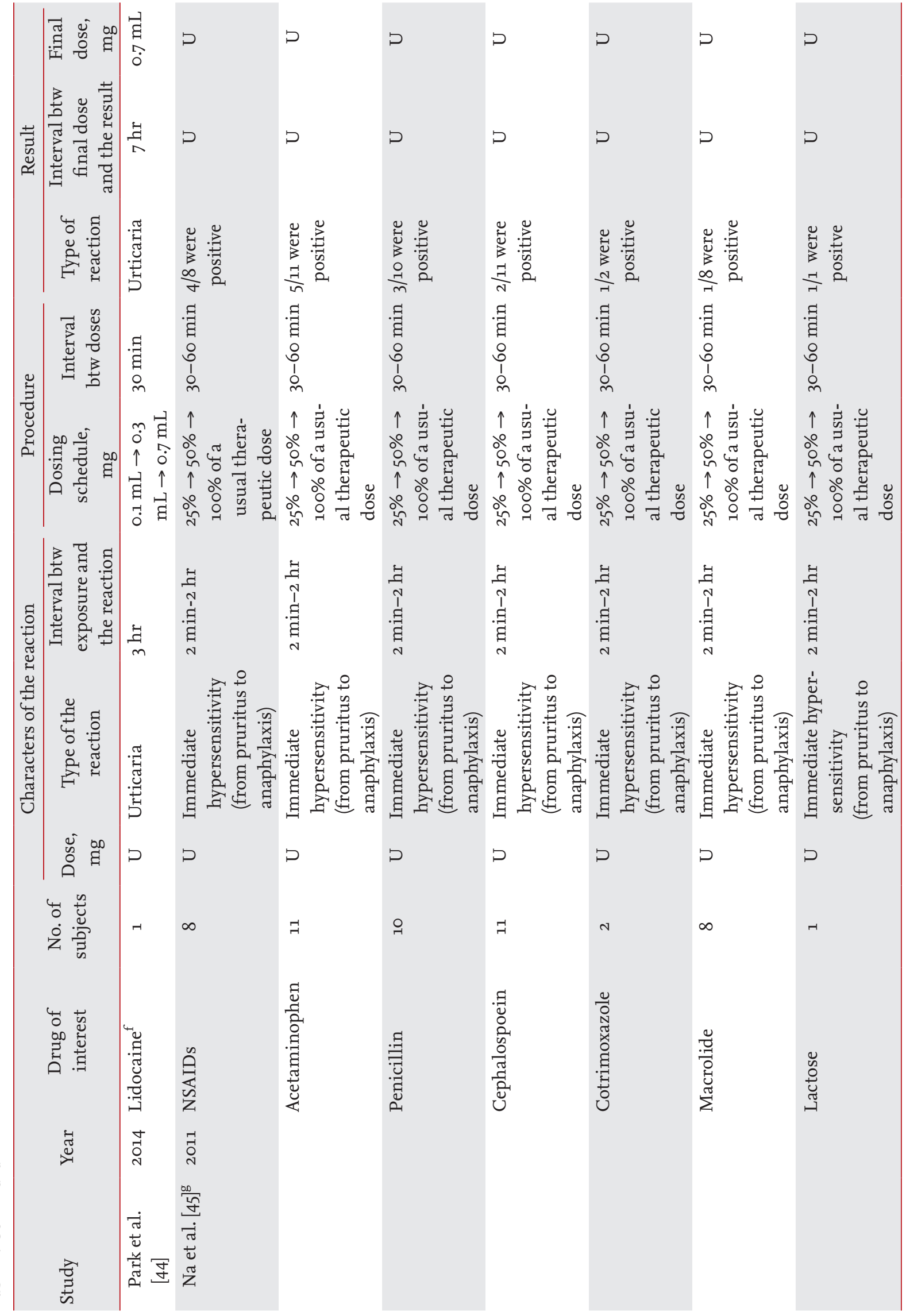



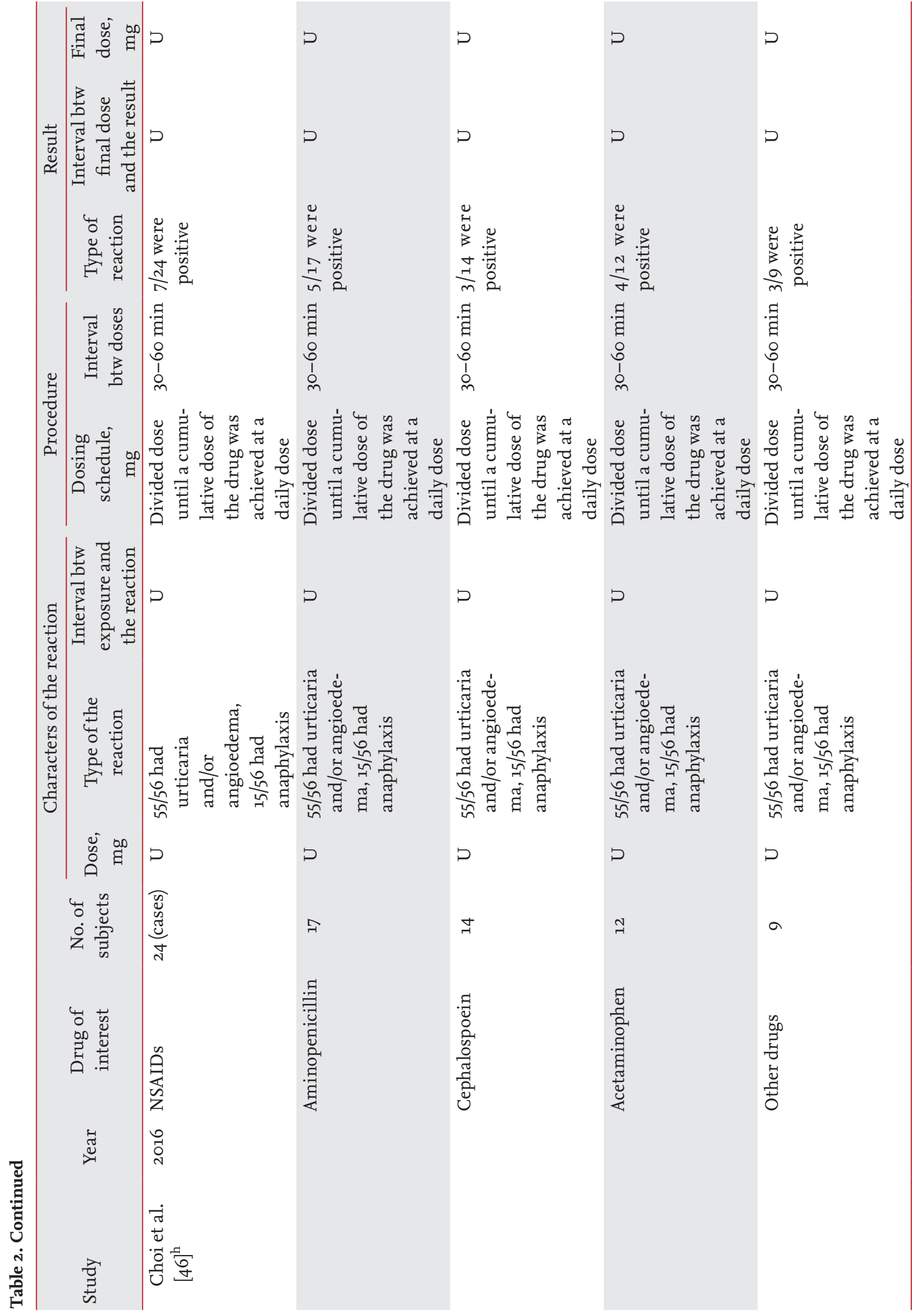


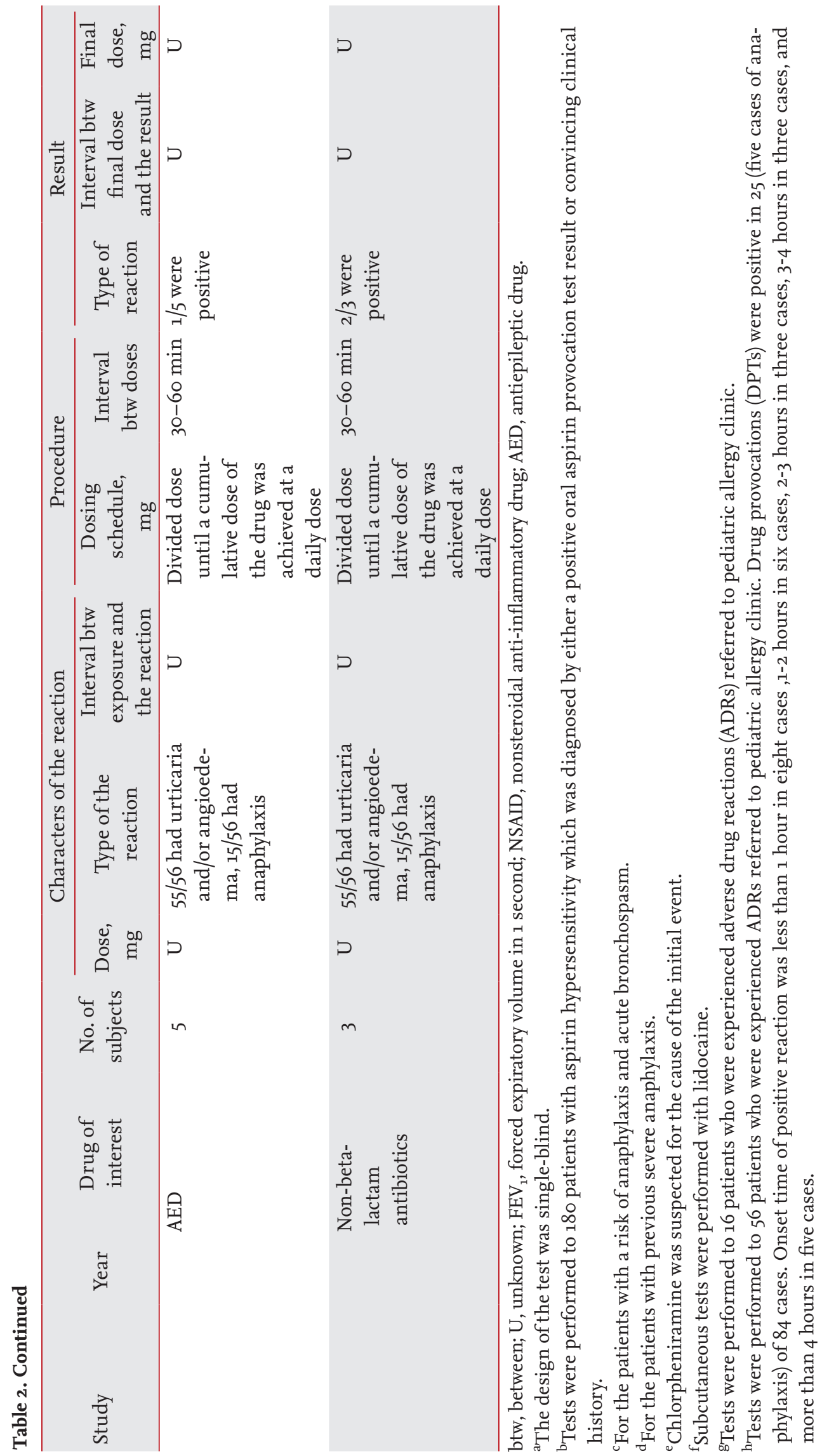


with aspirin usually followed the aspirin provocation test protocol from the EAACI/the Global Allergy and Asthma European Network (GA2LEN) guidelines with some modification [47].

\section{DISCUSSION}

This study presents the protocols for STs and DPTs from work published in Korea. STs and DPTs have been widely used to identify the culprit drugs of immediate allergic reactions, including anaphylaxis. An average of 1o healthy controls were enrolled for STs in each study to determine the NIC of the test drugs, and a 1/10 dilution of the concentrations used in SPTs were generally used in IDTs. The initial DPT dose ranged from one quarter of the standard therapeutic dose to one standard dose. Positive reactions to DPTs usually occurred within twice the original latency period.

Based on the time interval between drug administration and the development of the reaction, previous guidelines and reports have defined immediate or non-immediate DHRs using an arbitrary cut-off duration of 1 hour $[48,49]$. However, this classification remains controversial because IgE-mediated reactions can appear up to 6 hours after drug administration [11,50]. Under these circumstances, our study used a new proposed cut-off point: DHRs were classified as immediate when appearing within 1 to 6 hours of drug administration [12].

STs are generally considered safe and are frequently used to evaluate a culprit drug for immediate hypersensitivity. The chemical nature of the drug itself may elicit a false-positive reaction, and thus the results from normal controls must be reported together with those from the patients when determining the NIC $[6,51]$. Hence, finding the NIC is important for reliable STs, although the NICs of only a few drugs are known. Recently, the ENDA/EAACI Drug Allergy Interest Group reviewed articles written in English, German, Italian, French, and Spanish on the NICs of drugs and presented the NICs of many drugs collated from those articles $[6,51]$. Similarly, we sought to share the NICs determined in Korean studies by searching the Korean literature. We selected only studies with negative controls. In previous reports, IDTs were usually conducted with drug dilution of $1 / 10$ or lower to avoid irritant reactions that could be misinterpreted as positive [6]. Our results align with previous research. We present the skin test concentrations for $\mathrm{H} 2$ receptor antagonists, local anesthetics, leukotriene receptor antagonists, antitussives, vitamin supplements, hormones, and antibiotics other than penicillin from the Korean literature that have not yet been reported or reported less frequently elsewhere.

The DPT is the gold standard diagnostic method to confirm a diagnosis of drug hypersensitivity regardless of the underlying reaction mechanism. The basic principle of a DPT is to reproduce the hypersensitivity reaction in a controlled way. In 2003, the ENDA/EAACI Drug Allergy Interest Group published a position paper on DPT procedures [7]. They suggested that the test should be placebo-controlled. The suggested starting dose of the DPT should be between 1/10,oooth and 1/1oth of the therapeutic dose, dependent on the severity of the reaction in case of a previous immediate reaction. The time interval between doses should be at least 30 minutes. However, as this protocol requires substantial medical resources and time, it is unrealistic in some medical circumstances. In fact, the starting doses of DPTs in our study ranged from one quarter dose to a single therapeutic dose, and the interval between doses was 30 minutes in most reports. All DPTs in the studies we reviewed, except one involving a single-blind test, were open-label tests. Several other reports also used open-challenge tests regardless of a history of anaphylaxis [52-55]. In particular, one- or two-step DPTs with amoxicillin for de-labeling were conducted in patients with alleged penicillin allergy with or without preceding penicillin STs [53]. Moreover, one- or two-step DPTs with several drugs were proven to be as safe as multistep challenges in a select group of patients [55]. In this review, we found that positive reactions to all DPTs, except for one with levodropropizine, occurred at drug concentrations ranging from a half dose to the same dose as that used originally. These results suggested that one- or two-step DPTs can be performed safely in most cases. Furthermore, one- or two-step DPTs would not raise concern for tolerance induction [55]. As other researchers performed tests in a similar manner, multistep DPTs with aspirin or other nonsteroidal anti-inflammatory drugs (NSAIDs) were conducted for patients in Korea with suspected NSAID hypersensitivity [32,54]. 
Patients with NSAID hypersensitivity were challenged with aspirin instead of the drug that caused the original reaction. Hence, a variety of unexpected reactions can occur. Most of the reactions in these studies occurred within 60 minutes after administering the final DPT dose as the provocative dose. An interval of 30 to $60 \mathrm{~min}$ utes may be appropriate for most reactions. Although it has not been established how long patients should be monitored after the final dose is administered, most reactions occurred within twice the interval between drug exposure and the original reaction. Therefore, twice the interval between drug exposure and the original reaction should be sufficient for monitoring patients.

In this study, anaphylaxis is the most common reason for performing DPTs. Although DPTs pose significant risks for the recurrence of anaphylaxis in such cases, DPTs are usually needed to identify the culprit agent because DPTs are the gold standard for diagnosing anaphylaxis. It is often less dangerous to expose the patient to a suspected culprit agent in a controlled way than to fail to identify the culprit agent causing anaphylaxis. Note, however, that all DPTs included in our study were conducted by a trained allergy specialist with emergency resuscitation equipment and full monitoring of the patient. DPTs should not be performed by anyone other than an allergy specialist.

We acknowledge several limitations of this review. First, we reviewed DHR diagnostic procedures in journals published in Korea using the domestic KoreaMed search engine. However, our methodology was neither typical nor validated. Thus, the selection of articles analyzed might not be comprehensive. Second, many researchers give priority to publishing significant results, which may result in publication bias. The under-reporting of negative and inconclusive results may affect the interpretation of results in a review. Therefore, it was not clear whether the results really represented real-world clinical practice. Third, when determining the NICs of drugs, the numbers of control subjects were relatively small. Although the ENDA/EAACI position paper recommended that there be at least 20 healthy controls [6], we included studies with at least three healthy controls because we acknowledge that it is difficult to include more than 20 healthy controls in a clinical setting. Finally, the NIC of each drug presented here is a proposed concentration; that does not mean that the ST for each drug was valid. Nevertheless, the major strength of this review is that it shares quality articles published in a non-English speaking country with other parts of the world.

In conclusion, both STs and DPTs need to be standardized. Because few studies have examined the standardization of DHRs, efforts to share quality articles in different languages should be sustained to improve drug allergy testing.

\section{KEY MESSAGE}

1. Skin and drug provocation tests have been widely used to identify immediate allergic reactions to various drugs other than reported drugs.

2. Our report shares quality articles on the diagnosis of drug hypersensitivity reactions published in a non-English speaking country with other parts of the world. Efforts for standardizing diagnosis are required.

\section{Conflict of interest}

No potential conflict of interest relevant to this article was reported.

\section{Acknowledgments}

This research was supported by a fund (A092076) by Research of Korea Centers for Disease Control and Prevention.

\section{REFERENCES}

1. Vervloet D, Durham S. Adverse reactions to drugs. BMJ 1998;316:1511-1514.

2. Gomes ER, Demoly P. Epidemiology of hypersensitivity drug reactions. Curr Opin Allergy Clin Immunol 2005:5:309-316.

3. Thong BY, Tan TC. Epidemiology and risk factors for drug allergy. Br J Clin Pharmacol 2011;71:684-700.

4. Romano A, Torres MJ, Castells M, Sanz ML, Blanca M. Diagnosis and management of drug hypersensitivity reactions. J Allergy Clin Immunol 2011;127(3 Supp1):S67-S73.

5. Mayorga C, Fernandez TD, Montanez MI, Moreno E, 
Torres MJ. Recent developments and highlights in drug hypersensitivity. Allergy 2019;74:2368-2381.

6. Brockow K, Garvey LH, Aberer W, et al. Skin test concentrations for systemically administered drugs: an ENDA/ EAACI Drug Allergy Interest Group position paper. Allergy 2013;68:702-712.

7. Aberer W, Bircher A, Romano A, et al. Drug provocation testing in the diagnosis of drug hypersensitivity reactions: general considerations. Allergy 2003;58:854-863.

8. Torres MJ, Romano A, Celik G, et al. Approach to the diagnosis of drug hypersensitivity reactions: similarities and differences between Europe and North America. Clin Transl Allergy 2017;7:7.

9. Yang MS, Ban GY, Kim MH, et al. KAAACI Standardization Committee Report on the procedures and applications of the diagnostic tests for drug allergy. Allergy Asthma Respir Dis 2017;5:239-247.

10. Kim YY. Past, present, and future of allergy in Korea. Allergy Asthma Immunol Res 2010;2:155-164.

11. Bircher AJ, Scherer Hofmeier K. Drug hypersensitivity reactions: inconsistency in the use of the classification of immediate and nonimmediate reactions. J Allergy Clin Immunol 2012;129:263-264.

12. Demoly P, Adkinson NF, Brockow K, et al. International Consensus on drug allergy. Allergy 2014;69:420-437.

13. Lee YD, Cho Y, Han MS. Anaphylaxis due to ribostamycin. Allergy 2004;59:1134-1135.

14. Kim SG, Lee BH, Ryu JW, Shin SY, Kim SH. A case of hypersensitivity reaction to gemifloxacin. Korean J Asthma Allergy Clin Immunol 2006;26:159-162.

15. Lee KH, Choi IS, Lee WJ, Kang SJ, Jeong HK. A case of cefotiam-contact anaphylaxis. Korean J Med 2009;76(Suppl 1):S238-S242.

16. Kim SM, Kim KS, Kim MK. A case of anaphylaxis to trimethoprim-sulfamethoxazole. Korean J Med 2011;80:365369.

17. Koh YI, Park HS, Choi IS. Ranitidine-induced anaphylaxis: detection of serum specific IgE antibody. Allergy 2006;61:269-270.

18. Kim YI, Park CK, Park DJ, Wi JO, Han ER, Koh YI. A case of famotidine-induced anaphylaxis. J Investig Allergol Clin Immunol 2010;20:166-169.

19. Cho HJ, Yoo HS, Park SY, et al. A case of cimetidine-induced immediate hypersensitivity. J Investig Allergol Clin Immunol 2012;22:216-218.

20. Park KH, Pai J, Song DG, et al. Ranitidine-induced ana- phylaxis: clinical features, cross-reactivity, and skin testing. Clin Exp Allergy 2016;46:631-639.

21. Lee SM, Song WJ, Yang MS, et al. A case of lidocaine anaphylaxis. Korean J Asthma Allergy Clin Immunol 2006;26:249-253.

22. Koh YI, Choi IS, Lee HC, Na HS, Oh ST. Desensitization to urine-derived gonadotropins in a woman with secondary infertility. Ann Allergy Asthma Immunol 2001;87:434438.

23. Cho YS, Yoon TY, Choi W, Kim KS, Kim MK. A case of anaphylaxis induced by polysorbate 80 in parenteral multivitamin. Korean J Asthma Allergy Clin Immunol 2009;29:204-207.

24. Lee SY, Kim JY, Kang MG, et al. A case of anaphylaxis occurred after fluorescein angiography. Korean J Asthma Allergy Clin Immunol 2011;31:223-225.

25. Kim J, Park MR, Kim DS, et al. IgE-mediated anaphylaxis and allergic reactions to idursulfase in patients with Hunter syndrome. Allergy 2013;68:796-802.

26. Yoo HS, Yang EM, Kim MA, et al. A case of codeine induced anaphylaxis via oral route. Allergy Asthma Immunol Res 2014;6:95-97.

27. Kim S, Lee JM. A case of pranlukast-induced anaphylactic shock. Allergy Asthma Immunol Res 2016;8:276-278.

28. Jang JW, Bae YJ, Kim YG, et al. A case of anaphylaxis to oral minocycline. J Korean Med Sci 2010;25:1231-1233.

29. Kim SY, Choi GS, Kim HK, et al. Delayed onset urticaria and angioedema caused by components of itraconazole solution. Allergy Asthma Respir Dis 2014;2:138-141.

30. Lee YJ, Lee YW, Park JW, Hong CS. A case of propyphenazone induced asthma without aspirin sensitivity. Korean J Asthma Allergy Clin Immunol 2006;26:318-321.

31. Lee SH, Jung HS, Yoon TY, Chang EJ, Kim MK, Kim KS. Allergic reaction to chlorpheniramine in a patient with aspirin-intolerant asthma. Korean J Asthma Allergy Clin Immunol 2010;30:55-58.

32. Kim YJ, Lim KH, Kim MY, et al. Cross-reactivity to acetaminophen and celecoxib according to the type of nonsteroidal anti-inflammatory drug hypersensitivity. Allergy Asthma Immunol Res 2014;6:156-162.

33. Kim H, Yun SH, Kim MH, et al. A case of ranitidine-induced anaphylaxis. Korean J Asthma Allergy Clin Immunol 2010;30:140-143.

34. Lee SJ, Han YJ, Lee HW, Kim HK, Choi GS. A case of urticaria and angioedema induced by tiropramide. Korean J Asthma Allergy Clin Immunol 2011;31:300-303. 
35. Lee SY, Kim MY, Kang SY, Song WJ, Kang HR. A case of trimebutine-induced anaphylaxis. Allergol Int 2011;60:555566.

36. Choi SW, Han JM, Bae YJ, et al. Lessons from two cases of anaphylaxis to proton pump inhibitors. J Clin Pharm Ther 2012;37:614-616.

37. Jang EJ, Jin HJ, Nam YH, Kim JH, Ye YM, Park HS. Acute urticaria induced by oral methylprednisolone. Allergy Asthma Immunol Res 2011;3:277-279.

38. Lee SW, Byun JY, Choi YW, Myung KB, Choi HY. Fexofenadine-induced urticaria. Ann Dermatol 2011;23(Suppl 3):S329- $\mathrm{S}_{332 .}$

39. Hur GY, Hwang EK, Moon JY, et al. Oral muscle relaxant may induce immediate allergic reactions. Yonsei Med J 2012;53:863-865.

40. Kim SH, Lee J, Kim SH, et al. Anaphylaxis caused by muscle relaxant (eperisone hydrochloride). Allergy Asthma Respir Dis 2013;1:172-175.

41. Park KH, Yun IS, Choi SY, Lee JH, Hong CS, Park JW. A case of immunoglobulin E mediated anaphylaxis to levodropropizine. Yonsei Med J 2013;54:262-264.

42. Kim YU, Lee J. Drug hypersensitivity to various antihistamines with cross-reactions. Allergy Asthma Respir Dis 2014;2:134-137.

43. Yoon K, Kim SH, Ahn Y. A case of levodropropizine-induced anaphylaxis. Korean J Asthma Allergy Clin Immunol 2011;31:219-222.

44. Park GM, Han HW, Kim JY, et al. Delayed urticaria caused by lidocaine in a child. Allergy Asthma Respir Dis 2014;2:298-301.

45. Na HR, Lee JM, Jung JW, Lee SY. Usefulness of drug provocation tests in children with a history of adverse drug reaction. Korean J Pediatr 2011;54:304-309.

46. Choi J, Lee JY, Kim KH, Choi J, Ahn K, Kim J. Evaluation of drug provocation tests in Korean children: a single center experience. Asian Pac J Allergy Immunol
2016;34:130-136.

47. Nizankowska-Mogilnicka E, Bochenek G, Mastalerz L, et al. EAACI/GA2LEN guideline: aspirin provocation tests for diagnosis of aspirin hypersensitivity. Allergy 2007;62:1111-1118.

48. Romano A, Blanca M, Torres MJ, et al. Diagnosis of nonimmediate reactions to beta-lactam antibiotics. Allergy 2004;59:1153-1160.

49. Terrados S, Blanca M, Garcia J, et al. Nonimmediate reactions to betalactams: prevalence and role of the different penicillins. Allergy 1995;50:563-567.

50. Torres MJ, Salas M, Ariza A, Fernandez TD. Understanding the mechanisms in accelerated drug reactions. Curr Opin Allergy Clin Immunol 2016;16:308-314.

51. Joint Task Force on Practice Parameters; American Academy of Allergy, Asthma and Immunology; American College of Allergy, Asthma and Immunology; Joint Council of Allergy, Asthma and Immunology. Drug allergy: an updated practice parameter. Ann Allergy Asthma Immunol 2010;105:259-273.

52. Ramsey A, Mustafa SS. A penicillin skin testing initiative in an outpatient allergy office. J Allergy Clin Immunol Pract 2018;6:1756-1757.

53. Tucker MH, Lomas CM, Ramchandar N, Waldram JD. Amoxicillin challenge without penicillin skin testing in evaluation of penicillin allergy in a cohort of Marine recruits. J Allergy Clin Immunol Pract 2017;5:813-815.

54. Chaudhry T, Hissaria P, Wiese M, Heddle R, Kette F, Smith WB. Oral drug challenges in non-steroidal anti-inflammatory drug-induced urticaria, angioedema and anaphylaxis. Intern Med J 2012;42:665-671.

55. Iammatteo $\mathrm{M}$, Blumenthal $\mathrm{KG}$, Saff R, Long AA, Banerji A. Safety and outcomes of test doses for the evaluation of adverse drug reactions: a 5-year retrospective review. J Allergy Clin Immunol Pract 2014;2:768-774. 